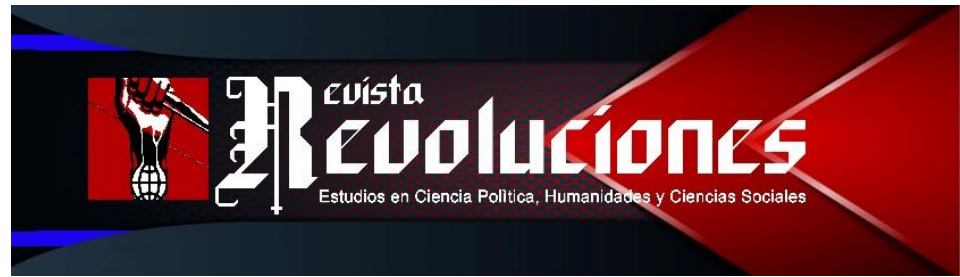

\title{
LOS ENFOQUES DEL TERCER MUNDO AL DERECHO INTERNACIONAL: UN ANÁLISIS DE LOS PRINCIPALES CONVENIOS SOBRE DERECHOS HUMANOS SUSCRITOS POR COLOMBIA DESDE LA ÓPTICA DE LOS TWAIL (1984-2018)
}

\section{The Third World approaches to International Law: an analysis of Colombia's main conventions on Human Rights from the perspective of TWAIL (1984-2018)}

\author{
Jorge Diego Mejía ${ }^{1}$ \\ UNIVERSIDAD DE ANTIOQUIA \\ COLOMBIA \\ jorged.mejia@udea.edu.com \\ https://orcid.org/o0oo-0002-3438-2401
}

DOI: https://doi.org/10.35622/j.rr.2020.02.002

Recibido: 02-I-2020 / Aceptado: 28-VI-2020 / Actualizado: 20-I-2021

\section{Resumen}

Las aproximaciones al Tercer Mundo desde el Derecho Internacional (TWAIL) son una serie de teorías o enfoques que pretenden buscar opciones a la forma eurocéntrica y colonialista como se han abordado tradicionalmente las relaciones internacionales. Colombia, al ser un país latinoamericano y tercermundista se encuentra doblemente condicionado a las políticas con las cuales se ha manejado el rumbo histórico de los pueblos. El presente estudio pretende realizar una aproximación a los convenios y tratados suscritos por Colombia desde 1984 hasta el 2018 en materia de Derechos Humanos desde la óptica TWAIL, determinar si se han cumplido y cómo dichos tratados y convenios han afectado o favorecido las Relaciones Internacionales.

Palabras Clave: TWAIL, Tercer Mundo, Derecho Internacional, Relaciones Internacionales de Colombia, Derechos Humanos.

\footnotetext{
${ }^{1}$ Coordinador de la Tertulia Literaria de Estudiantes Universidad de Antioquia. Normalista Superior, Normal Superior de Envigado. Politólogo Universidad de Antioquia. Fue director de la Casa de la Cultura Julio Cesar García del Municipio de Fredonia 2008-2011. Docente Centro Educativo Rural Filo de los Pérez del Municipio de Sabanalarga Antioquia. 2015. Profesor de Ciencias Sociales, Filosofía y Ciencias Políticas del Colegio Manuel Mejía Vallejo 2012. Columnista de Al-poniente. Profesor de Seminario de Investigación y Trabajo de grado en la Facultad de Derecho y Ciencias Políticas en la Universidad de Antioquia 2018-2020. Actualmente labora en la Secretaría de Cultura de Antioquia en el Área de Bienes.
} 


\begin{abstract}
Third World Approaches to International World (TWAIL) are a series of theories or approaches that seek to find options to the Eurocentric and colonialist way as international relations have traditionally been approached. Colombia, being a Latin American and third world country, is doubly conditioned to the policies with which the historical direction of the peoples has been managed. This study aims to make an approach to the agreements and treaties signed by Colombia from 1984 to 2018 in the field of Human Rights from the TWAIL perspective, to determine if they have been complied with and how said treaties and agreements have affected or favored International Relations.
\end{abstract}

Keyword: TWAIL, Third World, International Law, International Relations of Colombia, Human Rights.

\title{
INTRODUCCIÓN
}

El Derecho consiste en tres reglas o principios básicos: vivir honestamente, no dañar a los demás y dar a cada uno lo suyo. "Es el arte de lo bueno y lo equitativo" Ulpiano (170 - 228 A. C).

Colombia es un país que se ha caracterizado por tener buenas relaciones internacionales y también por estar suscrito a innumerables tratados y convenios de toda índole, no obstante, el hecho de que existan dichos pactos o acuerdos no significa precisamente que se cumplan. De acuerdo con el título se pretende analizar, desde la óptica de los Enfoques del Tercer Mundo al Derecho Internacional (TWAIL) los principales convenios sobre Derechos Humanos suscritos por Colombia desde 1984 hasta 2018, dado que, según los datos oficiales, desde este último año Colombia no ha suscrito ningún otro convenio relevante en materia de Derechos Humanos, y, se parte desde 1984 porque es en este año, en el que se suscribe la Convención sobre la tortura y otros tratos o penas crueles inhumanos o degradantes, que en Colombia se adoptó bajo la ley 70 de 1986. Es decir, un año después de la toma del Palacio de Justicia por parte del movimiento guerrillero M19 y en cuya retoma a 'sangre y fuego' por parte del Ejército Nacional, hubo varios desaparecidos y algunos exguerrilleros fueron torturados y otros asesinados. Es conveniente revisar hasta dónde Colombia ha cumplido o no con la comunidad internacional en medio de un arduo conflicto armado, más aun, después de la firma del acuerdo de paz con las Farc-Ep, en 2016, su implementación y toda la estructura legal que supuso dicho acuerdo entre el Estado, el grupo armado y la comunidad internacional como garante. También es importante intentar dilucidar a partir de estos hallazgos en qué estado se encuentran las relaciones internacionales de Colombia.

Los Enfoques del Tercer Mundo al Derecho Internacional, mejor conocido por su acrónimo TWAIL, es una red dinámica, abierta y descentralizada de académicos del derecho internacional que presentan una visión crítica sobre el Tercer Mundo y sus relaciones con otras esferas de poder. Dentro de la concepción de los TWAIL, el "Tercer Mundo" se refiere a 
esa geografía sociopolítica expansiva y generalmente subordinada que, a mediados del siglo XX, llegó a ser vista como "no alineada", es decir, que no encajaba en la concepción capitalista de "libertad" ni tampoco pertenecía al bloque "comunista".

Hoy, sin embargo, el Tercer Mundo es una denominación que se aplica con frecuencia a los países desarrollados o en desarrollo, al "mundo poscolonial" o el Sur (Global). En nuestro orden globalizado, intensamente desigual, racista, xenófobo, patriarcal y ambientalmente precario, que enfrenta la proliferación del Sur en el Norte y la intromisión del Norte en el Sur, esta geografía sociopolítica (que nace como una minoría) podría caracterizarse hoy como "la mayor parte del mundo" tomado en cuenta el número de países y la cantidad de personas en situación de pobreza.

Dado que, a través de la historia han ocurrido diversas olas colonialistas, para efectos del presente, cuando se habla de Colonialismo se toma como referencia, el periodo de la era hispánica y luego la presencia estadounidense, momentos coyunturales que han marcado significativamente el pulso de las relaciones internacionales y los Derechos Humanos. Países como Filipinas o Palaos tienen un aspecto histórico y una tragedia en común con Latinoamérica, con el descubrimiento del Nuevo Mundo, la guerra Hispano estadounidense de 1899, que nace de un atentado de falsa bandera (como fue la explosión del acorazado Maine) y la posterior ocupación tiránica de Estados Unidos, impusieron a través de la doctrina Monroe condiciones desiguales de relación y cooperación.

Estos sucesos, junto con los Acuerdos de Bretton Woods, la guerra fría, la operación Cóndor (entre otros) marcaron a los pueblos del sur. Tras años de ocupación y saqueo nos queda el presidencialismo como modelo político, el dólar como moneda de referencia, el capitalismo como sistema imperante, el subdesarrollo, la pobreza y la codependencia de los grandes capitales públicos o privados. Desde la óptica de los postulados TWAIL es necesario deconstruir las relaciones entre el tercer y el primer mundo y construir una nueva política internacional que abogue por la preservación de la cultura, la tradición de los pueblos nativos y los derechos humanos.

Colombia ha sido un país que se ha caracterizado por tener una diplomacia sumisa y poco preparada, lo que le ha suscitado innumerables derrotas diplomáticas. Se hará un breve recuento de ello, partiendo del Tratado Pombo-Michelena, que sería rechazado por Venezuela. Como lo afirma López Zea (1988) "El 14 de diciembre de 1833 se firma entre Colombia y Venezuela el primer tratado de límites, el famoso Pombo-Michelena, aprobado por nuestro país un año después y afortunadamente rechazado por Venezuela. Y se dice que afortunadamente porque con relación a la delimitación actual en ese tratado la Nueva Granada hacía extensas concesiones a Venezuela, le endosaba toda la costa Guajira, desde el cabo de Chichivacoa hasta Punta Espada, además del Alto Orinoco, parte del Casiquiare y Rionegro" (Pág.252). 
Hasta el Esguerra-Bárcenas de 1928 entre Colombia y Nicaragua que dio lugar al Protocolo de 1930, en el que Colombia reconoce la soberanía de Nicaragua sobre la Costa de Mosquitos y las islas Mangle Grande y Mangle Chico, argumento que luego utilizó Nicaragua para reclamar el archipiélago de San Andrés, Providencia, Santa Catalina y su mar adyacente. Pasando por el Thompson-Urrutia de 1914, en el que se fijaron los límites con la naciente República de Panamá y se acordó la pírrica indemnización de 5 millones de dólares, que solo se hicieron efectivas en 1922 o el Protocolo de Rio de Janeiro, que puso fin al conflicto colombo-peruano pero que concluyó con la pérdida de una parte importante de territorio allende a la cuenca del río Amazonas y sus afluentes. El Régimen Imperial apeló al principio de Uti Possidetis para legitimar su potestad de facto sobre los territorios que hoy son jurisdicción de Manaos, en el noroccidente de Brasil.

En innumerables ocasiones, el destino de los pueblos, generalmente pueblos nativos, (endémicos) se ve mediado por acciones gubernamentales o transnacionales, que, por la razón que el derecho público (o privado) les otorga o por la fuerza de quien detenta el poder; desconocen la autonomía, la cosmogonía y el sentir de estas minorías (etnias o tribus). El pueblo Wayú por ejemplo, se extiende entre Colombia y Venezuela, a lo largo y ancho de la península de la Guajira, la mayoría de ellos no se siente ni de uno ni de otro país; se siente, simplemente Wayú, y para ellos sus costumbres ancestrales trascienden las fronteras físicas.

Del mismo modo sucede con los Tikuna o los Kakuas en la frontera con Brasil y Perú, o los Emberá-Kuna en el Darién. Los procesos políticos, las diversas sectas que se han instalado en sus territorios y las dinámicas culturales impuestas han terminado por socavar esta soberanía cultural y la han empobrecido, la han segmentado en intereses de mercado y en un concepto de nación que obedece a las prácticas coloniales y republicanas. Un constructo estatal.

La imposición de un Estado implica la utilización de elementos identitarios que le permiten crear sentimientos unívocos, tales como, un escudo, un himno, un ejército, una creencia religiosa, una lengua, etcétera, y más acorde a los tiempos actuales, elementos como: un equipo de futbol, un ritmo musical, una comida típica, un sentimiento nacionalista que pueda ser recogido a través de un partido político; pasiones que convoquen a la homogeneización del concepto, que llamen a la unidad, pero desde una visión homogénea y, por ende, conservadurista , que no se abra al espectro de la disertación.

$\mathrm{Al}$ respecto, afirma la doctora María Elena Erazo (2008) "los valores simbólicos y culturales que comparten hoy los individuos que habitan el territorio colombiano y les hacen imaginar que pertenecen a una comunidad, se configuran a partir de la década de los 80 del siglo XIX, porque en ese periodo ella se asume como una invención histórica, política y cultural, razón por la cual en esa época, y concretamente a partir de la emisión de la Constitución de 1886, se inserta una serie de dispositivos, como la lengua, la religión, la lengua, la historia, que harán posible interiorizar los valores simbólicos y culturales de la religión católica, que se asumen como los valores y símbolos de la nación” (Pág. 49) A este clamor, por el respeto de 
las comunidades, insertadas o enclavadas en territorios nacionales donde generalmente no son mayoría es por el que claman los postulados TWAIL.

\section{METODOLOGÍA}

La presente investigación toma como punto de partida (y de referencia) los conceptos básicos del Derecho Internacional. En primer lugar, es necesario determinar, (como en las demás ramas del Derecho) cuáles son las fuentes formales del Derecho internacional, que son, lógicamente, las fuentes materiales y las formales. Las primeras son, en palabras de M. Sorensen (1994) los factores que provocan la aparición de las normas y determinan su contenido; estos pueden ser de carácter ético, religioso o impuestos por la tradición, en tanto que, las fuentes formales, son aquellas que tienen un contenido obligatorio per se, y una naturaleza jurídica concreta.

Según Browlie (1995) las fuentes formales del Derecho Internacional (...) han sido enumeradas en dos tratados-leyes a saber, el primero: artículo séptimo del convenio 12 firmado en La Haya en 1907 y luego en el artículo 38-1 del Estatuto de la Corte Permanente de Justicia Internacional (CPJI) y de la Corte Internacional de Justicia, ambos bloques normativos podrían considerarse la génesis del Derecho Internacional, del cual beben y problematizan los TWAIL en sus vertientes principales: Derecho internacional público y el privado. En materia de Derechos Humanos, el primer y más significativo paso fue la Declaración Universal de Derechos Humanos que fue adoptada por la tercera Asamblea General de las Naciones Unidas, el 10 de diciembre de 1948 en París. Ninguno de los 56 miembros de las Naciones Unidas votó en contra del texto, aunque Sudáfrica, Arabia Saudita y la Unión Soviética se abstuvieron.

Posteriormente el estudio se focaliza, (en su primer grado de teorización) en los estudios críticos del derecho, no obstante toma prestados aspectos epistemológicos de los estudios decoloniales y en segundo grado (más concretamente en los enfoques tercermundistas del Derecho Internacional (TWAIL), que a su vez se desprenden de las llamadas NAIL (New Approaches to International Law) o Nuevas Aproximaciones al Derecho Internacional.

Cuando se analizan los fundamentos teóricos de los estudios decoloniales y tercermundistas se pueden intuir los principios filosóficos de los mismos a partir de sus teóricos. En este nicho epistémico coexisten dos disciplinas perfectamente diferenciables: la sociología y el derecho, de los cuales evolucionan otras disciplinas, primero la ciencia política y segundo el derecho internacional. De allí se desprenden la geopolítica y las relaciones internacionales. Sin embargo, Weber, uno de los precursores del estudio de la sociología jurídica, intenta desarrollar a partir de este campo epistemológico la microsociología, entendida esta como el estudio de los sistemas sociales y poblacionales a gran escala y cuyo objeto de estudio se enfoca principalmente en la estructura social, y frecuentemente al nivel de la abstracción teórica clásica. 


\section{DESARROLLO}

Antes de abordar el planteamiento del problema y con base en la pregunta problematizadora, es necesario realizar un preámbulo acerca del tema en cuestión presentado en tres estadios: en el preámbulo se hará una aproximación a los antecedentes del Derecho Internacional y las relaciones internacionales, en la primera parte se intentará responder a la pregunta: ¿Qué se entiende por Tercer Mundo? Luego, en un segundo momento ¿̇qué se entiende por TWAIL? y ¿qué se entiende por Derecho Internacional? Por último, una aproximación a las relaciones internacionales de Colombia, especialmente lo que tiene que ver con Derechos Humanos intervenidos desde la óptica TWAIL.

Como preámbulo, que configura la legislación constitutiva del Derecho Internacional que hoy se aplica a los países agremiados. Tenemos entonces, como antecedentes del Derecho Internacional, en primer lugar, la Paz de Westfalia que supone el primer congreso diplomático moderno, que se deriva de los tratados de paz de Osnabrück y Münster. Luego, en 1914 se produce la guerra entre serbios y austriacos y que desemboca en la primera guerra mundial, que culmina en 1918 cuando Alemania acepta las condiciones del armisticio. Con el tratado de Versalles se crea la Sociedad o Liga de las Naciones, como aproximación primigenia a un estamento de orden internacional. Por último, vendrían la Carta de San Francisco firmada el 26 de junio de 1945 después de que finaliza la segunda guerra mundial.

Este acontecimiento es quizá el más relevante para el Derecho Internacional Público; pues el Derecho Privado se configura de un modo diferente, un enfoque netamente crematístico. Con la consolidación de las Naciones Unidas y el desarrollo de las Relaciones Internacionales se afianzaría el Derecho Internacional como carta magna que regula los diferentes procedimientos entre países y con esta se gesta un principio de regulación de dichas relaciones, no solo desde lo geopolítico, sino también desde la cooperación económica y social. La forma como Colombia se relaciona con el mundo desde sus inicios como república no proyecta precisamente un vector horizontal; $\mathrm{y}$ la forma transversal de relacionamiento no es un problema solo de Bogotá, sino del bloque latinoamericano y del resto de los países en situación de tercermundismo según los estándares cuantitativos de occidente.

Es cierto que el sur global fluctúa de manera distinta en cada continente, a pesar de tener cuestiones en común, Asia por ejemplo se mueve en una dinámica distinta a la de occidente, en el sudeste asiático el Respice Pollum ${ }^{2}$ (referente) que se impone tangencialmente desde las últimas décadas es China, a pesar de la influencia militar que ejerce aun Estados Unidos con sus múltiples bases en los principales archipiélagos del pacifico. TWAIL por ejemplo apuesta a cambiar ese Respice Pollum por un Respice Similia3, es decir, mirar a los que son

\footnotetext{
${ }^{2}$ El presidente Marco Fidel Suárez acuñó la doctrina del Respice polum o la mirada a la estrella polar (del Norte), haciendo explícita alusión de la subordinación ejemplificante a los Estados Unidos de América.

3 Mirar a los semejantes, relacionarse con sus pares
} 
semejantes, relacionarse con aquellos con los que se tienen características similares y adoptar una mirada horizontal, o al menos más humana de las relaciones internacionales.

Las relaciones internacionales han fluctuado entre el idealismo y el realismo político, pero en los últimos años se han visto posturas neorrealistas, combinadas con un proteccionismo económico sin precedentes, a manos de outsiders políticos como Trump, además del avance de la ultraderecha en Europa. Estas tendencias no son ajenas al tercer mundo y llegan como reflejo y como ejemplo, a través de políticas importadas desde las embajadas y consulados o desde las recomendaciones económicas del FMI o del Banco Mundial. El problema y a la vez el desafío es: ¿Cómo desligarse de esos nexos históricos de dominación y establecer unas relaciones internacionales que respeten los derechos humanos, sin perder la soberanía? El reto de Colombia es inmenso, ahora que pertenece a la OCDE4, a PROSUR, a la CAN, que es Socio global de la OTAN y de Mercosur, pero también es un reto interesante, por el costo político y económico que ello conlleva. No obstante, deberá subsanar la inmensa y creciente brecha de desigualdad, resolver un conflicto interno que aún pervive, una alta tasa de corrupción y mejorar unas condiciones de infraestructura social, gobernanza y gobernabilidad, si desea encumbrarse en la élite económica mundial. Reto que ni siquiera Chile o Brasil han podido lograr, máxime en tiempos de Covid-19.

\section{¿Qué se entiende por Tercer Mundo?}

El término tercer mundo fue acuñado por el economista francés Alfred Sauvy en 1952, quien pretendía hacer una analogía con el término "Tercer Estado" que en la Francia de la revolución describía la población carente de privilegios jurídicos y económicos, por tanto, se utilizó el término para designar a los países que no pertenecían a ninguno de los dos bloques enfrentados en la Guerra Fría, el bloque occidental y el bloque comunista.

\section{¿Qué se entiende por TWAIL?}

Según J.M Barreto (1989) TWAIL (Third World Approaches to International Law) es una de las teorías recientes que más ha contribuido a repensar el paradigma vigente del Derecho Internacional. Siendo además una de las principales corrientes críticas del derecho internacional contemporáneo, TWAIL ha contribuido a dar un giro epistémico de la tendencia histórica que caracteriza al derecho internacional; puesto que replantea su visión netamente occidentalista. TWAIL ha desafiado el paradigma eurocéntrico del derecho internacional y lo ha redefinido al contextualizarlo en la historia del colonialismo moderno -desde la época de la Conquista de América hasta el momento actual de la globalización.

Los inicios de TWAIL como movimiento intelectual se remontan a la primera conferencia celebrada en la Universidad de Harvard en 1997 relacionada con el tema, donde se presentaron un importante número de papers y artículos que exploraban los contornos generales de su significativa producción intelectual. Estas circunstancias se basan en una

4 Organización para la Cooperación y el Desarrollo Económicos 
reflexión más sostenida sobre TWAIL que es ahora necesaria para mostrar toda su complejidad y el alcance de su contribución a una teoría general del derecho internacional, así mismo es necesario situar a los TWAIL en el panorama de la historia moderna y la teoría legal internacional contemporánea.

En este orden de ideas la proyección de los TWAIL no se basa en crear un nuevo orden mundial, sino en fomentar la descolonización y crear mecanismos de acceso igualitarios del tercer mundo en las relaciones internacionales, no desde la óptica de la sumisión, sino desde la diplomacia de la hermandad y la geopolítica de la equidad; especial y fundamentalmente en lo que concierne a Derechos Humanos.

\section{Aproximación a las Relaciones Internacionales de Colombia}

El 15 de febrero de 1819, durante el Congreso de Angostura5, fue proclamado el Estado que adoptó el nombre de República de Colombia, pero que es históricamente conocido como Gran Colombia. Sin embargo, Colombia se constituye nominalmente como "República de", a partir de la constitución de 1886 que fue derogada por la constitución de 1991. La historia de las relaciones internacionales se remonta al periodo independentista, cuando en 1830 rompe relaciones con Madrid y las establece con Londres y Paris. En palabras de Carrera Damas (1930), "El Pacto de la Unión, celebrado el 20 de septiembre 1861 establece en su artículo primero los conceptos de "soberanía" e "independencia" para los Estados recién creados y sus autonomías, además de las obligaciones que tienen con los demás Estados de la Unión de ayuda y socorro mutuo en caso de guerra ya sea viniendo de otro país o de otro Estado de la unión". Actualmente, una de las condiciones fundamentales para que una nación sea cobijada por el Derecho Internacional es el reconocimiento de cierto número de naciones.

En la historia contemporánea, con el final de la segunda guerra mundial y el inicio de un nuevo orden mundial, Colombia, que había declarado el "estado de beligerancia" contra Alemania en $1943^{6}$, firma la Carta de San Francisco, el 24 de octubre de 1945, y cuya firma supone la inclusión automática de nuestro país a la Organización de Naciones Unidas, conforme lo dicta el Capítulo II referido a miembros:

\footnotetext{
"Son miembros originarios de las Naciones Unidas los Estados que, habiendo participado en la conferencia de las Naciones Unidas celebrada en San Francisco, o que habiendo firmado previamente la declaración de las Naciones Unidas del 1 de enero de 1942, suscriben esta carta y la ratifiquen de conformidad con el artículo $110^{7}$."
}

\footnotetext{
5 El Congreso se instaló por orden del General Simón Bolívar con el objetivo de formular la "Ley Fundamental", insumo primigenio de la Constitución Nacional

${ }^{6}$ La decisión la toma el entrante presidente Darío Echandía tras el hundimiento en el mar Caribe de las goletas colombianas Resolute, Roamar y Ruby por submarinos nazis.

7 Fuente: Ministerio de Relaciones Exteriores de la Republica de Colombia. Cancillería. Recuperado de: http://apw.cancilleria.gov.co/Tratados/adjuntosTratados/FC3F4_ONU-1945.PDF
} 
A continuación, se relaciona un cuadro con los principales Acuerdos Internacionales sobre Derechos Humanos y Derecho Internacional Humanitario, firmados por Colombia con la Comunidad Internacional, según los datos oficiales de la Cancillería:

\section{Tabla 1. Acuerdos Internacionales de Colombia entre 1984 - 2018.}

Acuerdo mediante canje de notas entre la República de Colombia y las

$19 / 03 / 2018$

Naciones Unidas, representadas por la Entidad de las Naciones Unidas para la igualdad de género y el empoderamiento de las mujeres (ONU mujeres) sobre la presencia de la ONU mujeres en Colombia

Establecimiento en Colombia de una Oficina del Alto Comisionado de las Naciones Unidas para los Derechos Humanos, firmado en Ginebra el 29 de noviembre de 1996

Convención Internacional para la Protección de todas las personas contra las desapariciones forzadas

Convención sobre los derechos de las personas con discapacidad $18 / 07 / 2013$ Protocolo para prevenir, reprimir y sancionar la trata de personas, especialmente mujeres y niños, que complementa la Convención de las Naciones Unidas contra la delincuencia organizada transnacional Protocolo facultativo de la Convención sobre los Derechos del Niño relativo 25/05/2000 a la participación de niños en los conflictos armados Protocolo facultativo de la Convención de las Naciones Unidas para la eliminación de todas las formas de discriminación contra la mujer Convención interamericana para la eliminación de todas formas de discriminación contra las personas con discapacidad Estatuto de Roma de la Corte Penal Internacional $20 / 12 / 2006$ Convención Interamericana para prevenir, sancionar y erradicar la violencia contra la mujer "Convención de Belem do para" en materia de Derechos Económicos, Sociales y Culturales "Protocolo de San Salvador"

Convención sobre la tortura y otros tratos o penas crueles inhumanos o 20/11/1989 $17 / 11 / 1988$ degradantes

Fuente: elaboración propia a partir de la fuente: Ministerio de Relaciones Exteriores. Cancillería. Recuperado de:

http://gruposmre.cancilleria.gov.co/sitios/DVAM/DIDHD/Biblioteca/SitePages/Instrumentos\%2oInternacio nales.aspx

Antes de la Convención de 1984, Colombia firma una serie de convenciones que, aunque relevantes, no entrarán a ser parte del presente estudio. (Ver justificación) En primer lugar, la Convención Interamericana sobre Concesión de Derechos Civiles a la Mujer de 1948. En 1966 la Convención Internacional de las Naciones Unidas sobre la eliminación de todas las 
formas de Discriminación Racial y en 1969 La Convención Americana sobre Derechos Humanos: Pacto de San José de Costa Rica.

La Convención de 1984 es un tratado que se incorpora al sistema jurídico colombiano bajo la Ley 70 de 1986 (DICIEMBRE 15) por medio de la cual se aprueba la "Convención contra la tortura y otros tratos o penas crueles, inhumanos o degradantes", adoptado en Naciones Unidas el 10 de diciembre de 1984. Nota: Convención promulgada por el Decreto 768 de 1988. Dicho decreto se firma junto con un grupo de convenios y acuerdos de diferentes fechas (1985-1987) y que tratan de diferentes asuntos, desde el Acuerdo de Cooperación de Cartagena y sus países miembros, Bolivia, Colombia, Ecuador, Perú y Venezuela, hasta el Acuerdo de Cooperación Técnica y Científica entre el Gobierno de la República de Costa de Marfil y el Gobierno de la República de Colombia", suscrito en Abidjan el 10 de agosto, de 1984. Tan solo en el Artículo 9 se menciona dicha Convención: $9^{\circ}$. "Declárase vigente para Colombia desde el 7 de enero de 1988 la "Convención contra la Tortura y otros tratos o penas crueles, inhumanos o degradantes", hecha en New York el 10 de diciembre de 1984. Firman el presidente de la República Virgilio Barco Vargas y el Ministro de Relaciones Exteriores Julio Londoño Paredes. Por su parte, el documento oficial de la ONU emitido en 1985 se codifica con el numero Ch_IV_9. En la redacción de este se invoca en primera instancia el Artículo 55 de la carta de las Naciones Unida. Exhortando a la obligación que 
incumbe a los Estados, de promover el respeto universal, la observancia de los Derechos Humanos y las libertades fundamentales.

Figura 1: elaboración propia con base en la investigación del anteproyecto.

\section{DESARROLLO}

El hecho de que la humanidad aún esté vivo en su mayoría y siga de pie, puede ser una de las grandes premisas del siglo XXI. La especie humana en su mayoría indigente, enfrentó cuerpo a cuerpo al satánico virus con sus propios medios ancestrales, a pesar de que necesitaron apoyo de sus respectivos actores gubernamentales, nunca tuvieron coherentemente una respuesta viable. Todos los centros de atención al paciente Covid-19 colapsaron

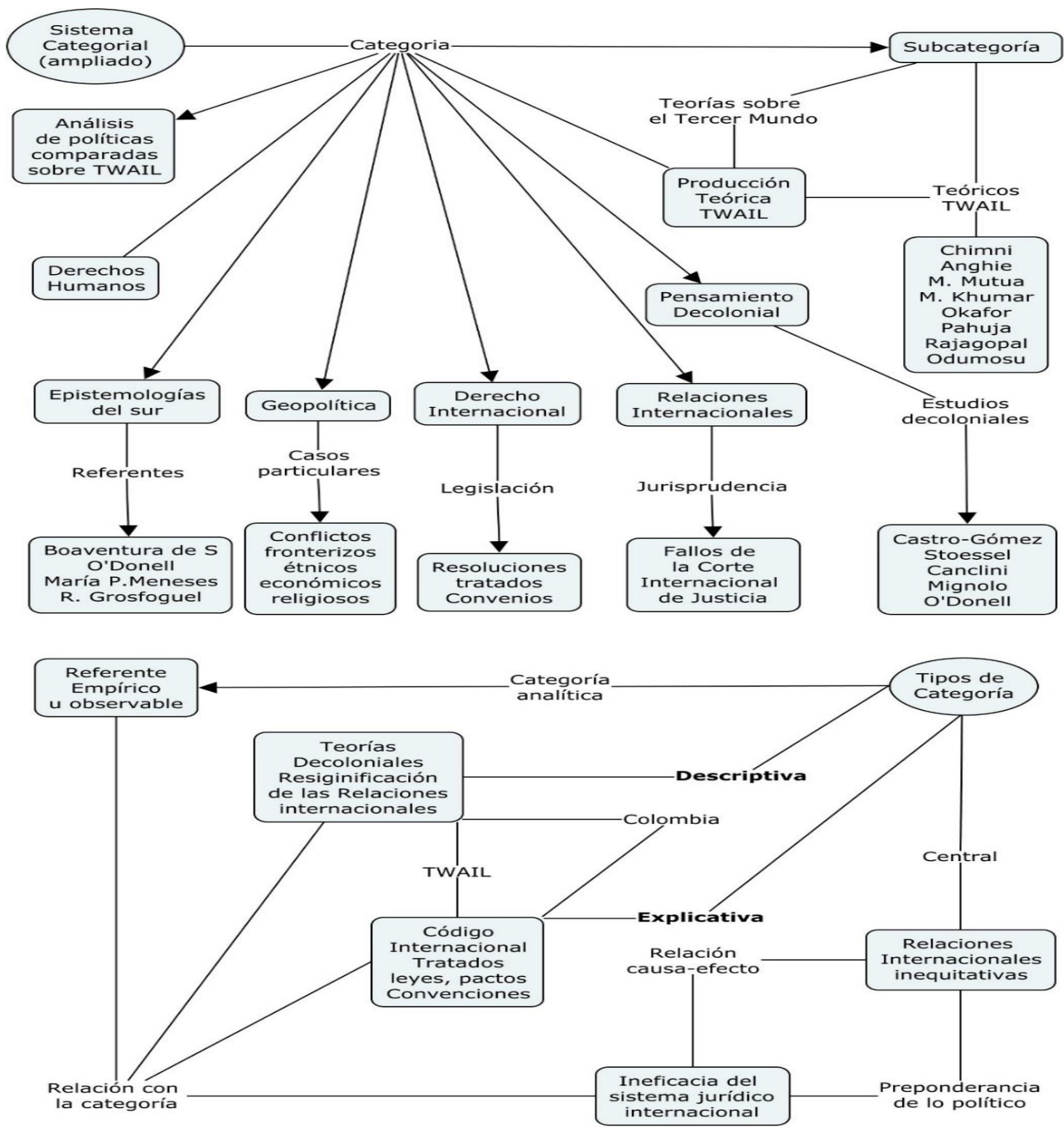

Revista Revoluciones $\mathbf{- 1 6}$ - Vol. 2, No 2 (2020), pp. 6-27

Esta obra está bajo una licencia internacional Creative Commons Atribución 4.0. 
sistemáticamente y el personal de salud fue impotente hacia esa enfermedad desconocida hasta la actualidad.

\section{Marco teórico de la investigación}

Para Fariñas (1988) la consideración científico-sociológica del derecho utiliza un método empírico de análisis causal, que Weber desarrolla, tanto en el ámbito de la macrosociología, esto es cuando se propone investigar las causas y efectos de la existencia fáctica de un orden jurídico, como en el ámbito de la microsociología, es decir, cuando se propone la investigación del origen y las causas de determinadas regularidades empíricas, calificables como jurídicas. Por el contrario, la aproximación dogmático-jurídica utiliza un método lógico normativo de carácter formal, que trabaja con el «contenido de sentido objetivo lógicamente correcto de proposiciones jurídicas», permaneciendo, por tanto, en el mundo de los conceptos. En cuanto a la finalidad de ambas aproximaciones científicas, la distinción es clara: la Sociología del Derecho intenta averiguar lo que sucede de hecho en la realidad, esto es, tratar de determinar cuál es el comportamiento de los miembros de una asociación o grupo, en relación con un orden jurídico en vigor. (p.208)".

Marc Galanter (2003) por su parte, opina que la mayoría de los análisis del sistema jurídico comienzan con las reglas y siguen con las entidades institucionales, para determinar qué efectos tienen las reglas sobre las partes. Su propósito era invertir el procedimiento y mirar desde el otro lado del telescopio. "Pensemos en los diferentes tipos de partes y en el efecto de estas diferencias en la manera cómo funciona el sistema” (p. 72) En un sistema global (o globalizado) los sistemas jurídicos locales (o endémicos) están supeditados a grandes y poderosas instituciones que poco o nada les importan las características culturales o vernáculas de algún pueblo, inmerso (Quizá) en medio de un estado e intervenido por intereses mercantilistas y/o extractivistas ${ }^{8}$. Es decir, lo global como un imperativo que consume, sustituye y oprime lo estatal. En eso consiste y consistió la avanzada neoliberal, que desconoció la riqueza e idiosincrasia de las naciones del tercer mundo.

Para Wallerstein (2006), pensar globalmente, actuar localmente, es un lema que muy deliberadamente excluye al Estado, y representa una suspensión de la fe en el Estado como mecanismo de reforma (...) Ambos foros de análisis (global y local) tomaban las incertidumbres y los localismos como variables analíticas centrales que no deberían ser enterradas en un universalismo determinista en consecuencia, la naturaleza evidente de los estados como contenedores conceptuales quedó abierta al cuestionamiento serio y al debate. Obviamente el pensamiento estadocentrista no había excluido el estudio de las relaciones entre los estados, o relaciones internacionales y dentro de cada una de las ciencias sociales existían subcampos dedicados al llamado espacio internacional. (p.90) Después de la segunda guerra mundial, con la consolidación de las potencias, la conformación de bloques y la guerra fría se instaura un nuevo orden mundial, las relaciones internacionales se ven mediadas por

8 Este tipo de investigaciones son adoptadas por los FWAIL (Aproximaciones al cuarto mundo desde el derecho internacional) 
el fantasma del colonialismo y nacen nuevos enfoques y teorías sobre el Derecho Internacional y su deber ser, basado en el pensamiento libertario.

En 1955 se llevó a cabo la conferencia de Bandung, en Indonesia, una reunión de estados asiáticos y africanos en su mayoría recién emancipados, cuyo propósito era mantenerse al margen de las potencias colonizadoras y crear una nueva forma de relacionarse con la región y con el mundo, de esta cumbre surgió el movimiento de los No Alineados (NOAL) países que querían tomar distancia, tanto del Bloque Comunista, como de la OTAN. Si bien el Derecho ha sido una herramienta emancipatoria, también ha servido muchas veces para crear ordenamientos jurídicos coercitivos; lo que ocurre en el ámbito de lo personal perfectamente puede replicarse en el ámbito regional o lo global. Como ciertamente lo afirmaba De la Torre (2007), podemos afirmar que, el Derecho puede ser usado políticamente. Y la política del Derecho puede constituir su uso alternativo. Es decir, usar el Derecho en contrasentido al papel asignado por el modelo de producción y distribución de bienes en la sociedad (p. 125). Efectivamente el uso que se le dio al derecho internacional, a partir de 1955 fue un uso político, frente a las voraces y desiguales relaciones internacionales que hasta el momento dominaban el mundo, muy lejos del idealismo político y muy cercano al realismo positivista.

Del espíritu de los Noal, se han desprendido varias propuestas ideológicas, académicas y políticas que han desembocado en el llamado movimiento TWAIL Las agendas académicas asociadas con TWAIL son diversas, pero el tema general de sus intervenciones es desempaquetar y deconstruir los legados coloniales del derecho internacional y participar en los esfuerzos de descolonización. El término fue acuñado en la década de 1990 a través de una alianza de académicos comprometidos a investigar críticamente la relación mutuamente constitutiva entre el derecho internacional, el Tercer mundo y el Sur global, para proyectos legales que operan al margen de la disciplina principal. La red TWAIL permite la solidaridad y el apoyo mutuo a través de un compromiso político compartido para abogar por los intereses del Sur global y sur-sur. Se esfuerza por dar voz a los diferentes puntos de vista de los países y pueblos sistemáticamente subrepresentados o silenciados.

El grupo se reunió por primera vez en Harvard Law School en 1997 y ha crecido rápidamente desde entonces, con conferencias en Osgoode Hall Law School en 2001, Albany Law School en 2007, University of British Columbia en 2008, Paris en 2010 y Oregon Law School en 2011 La conferencia de 2015 en la Universidad Americana del Cairo, convocó a la primera gran reunión de académicos TWAIL con el ánimo de debatir sobre el Sur global, establecer una bitácora para revisar eventos pasados de TWAIL y una proyección a futuro. La conferencia tuvo como objetivo proporcionar el espacio para que los académicos continúen colaborando y trabajando contra la desigualdad y en favor de los derechos humanos.

Es sabido que, a pesar de los avances tecnológicos y de vivir en una era llena de posibilidades, el ser humano y en especial las grandes potencias, tienen que lidiar con asuntos que denigran de la condición humana y que varían circunstancialmente de un grupo social a otro y aunque en la mayoría de los casos el ordenamiento jurídico de cada nación contempla dichas divergencias y aristas expresadas en la cultura, en numerosas ocasiones dicha intervención no es suficiente para que las poblaciones más vulnerables reciban una efectiva justicia social. 
Estados Unidos, que paulatinamente se ha querido constituir como el adalid de la democracia y el guardián del mundo, sufre hoy por hoy todas estas conductas. Su territorio es un crisol de culturas cuya convivencia no es siempre pacífica. Cuando Austin (2001) indagaba por las percepciones de la gente hacia el derecho en general y la percepción de la asistencia social en Norteamérica se encontraba con declaraciones como esta: "Si quieres saber la verdad, yo creo que los trabajadores sociales, los abogados y los jueces odian a los puertorriqueños. Ahora somos muchos y eso los pone a todos nerviosos porque desde su punto de vista, la asistencia está bien para los blancos, pero ayudarles a los puertorriqueños es igual que ayudarles a los animales (p.8)”.

Puerto Rico, sufre una extraña condición, es un estado libre asociado a la Unión americana, cuando en realidad no es "ni libre ni asociado" o en otras palabras, es para unas cosas libre y para otras asociado (a conveniencia de los legisladores estadounidenses); es un país que al igual que Filipinas, pasó de ser colonia española a colonia norteamericana, sin poder oponer mayor resistencia. A este tipo de prácticas precisamente se oponen los TWAIL, evocando los principios derivados de la conferencia de Bandung y los principios del Derecho Internacional.

En congruencia con el párrafo anterior y respecto a las proyecciones y lecciones que los estudios del tercer mundo puedan aportar a Latinoamérica, he rescatado este párrafo extraído de López (2004) quien aduce que, lo que comparten los diversos campos del pensamiento jurídico latinoamericano es un cierto ángulo de visión, una perspectiva de análisis que reconstruye y refuerza el lugar de América Latina como "contexto de recepción" de normas, teorías y doctrinas provenientes de los "contextos de producción" del Norte global, en general, y de los Estados Unidos, en particular (Rodríguez Garavito, 2011, p. 12) En otras palabras, si nuestros pueblos quieren alcanzar soberanía, armonía y cohesión, deben procurar por depender lo mínimo de la hegemonía del capital, buscar establecer una normativa internacional basada no en la ventaja sino en el reconocimiento, basado en unas reglas de juego justas, basadas en el bien común y no en intereses particulares.

Durkheim (2001) asentía al respecto: "pero no basta que haya reglas, es preciso, además, que sean justas, y para eso es necesario que las condiciones exteriores de la concurrencia sean iguales. Si, por otra parte, se recuerda que la conciencia colectiva se reduce cada vez más al culto por el individuo, se verá que lo que caracteriza la moral de las sociedades organizadas, comparada a la de las sociedades segmentarias, es que tiene algo de más humano, por consiguiente, de más racional. No hace depender nuestra actividad de fines que no nos tocan directamente; no hace de nosotros los servidores de poderes ideales y de naturaleza distinta a la nuestra, que siguen sus propios caminos sin preocuparse de los intereses de los hombres (p. 261)".

Respecto a lo que concierne propiamente al modelo TWAIL, como corriente de pensamiento derivada de los estudios críticos del Derecho, se reconocen varios autores, imprescindibles, pero no por ello incuestionables. El primero de ellos es el doctor José Manuel Barreto, que a 
partir de un ensayo titulado ¿Qué es TWAIL? Expone las razones epistemológicas del pensamiento TWAIL, en estas palabras:

"El proyecto de TWAIL intenta re-escribir la historia del derecho internacional desde la óptica de la historia del imperialismo apunta a una reelaboración sustancial del derecho internacional -del paradigma que ha estado vigente en los últimos siglos y que orienta su aplicación en el Siglo 21. De manera especialmente pertinente para nosotros, TWAIL no es solamente otra nueva concepción crítica del derecho internacional, sino que nos ofrece a los latinoamericanos, y a los ciudadanos del Tercer Mundo en general, la posibilidad de interpretar y usar el derecho internacional desde nuestro punto de vista. Es decir, desde la historia del mundo colonizado en la modernidad -o desde la perspectiva de los colonizados (J.M. Barreto, 2018)".

Luego viene B.S. Chimni con "Un manifiesto TWAIL" afirmando que: "La amenaza de recolonización está persiguiendo tercermundistas. El proceso de globalización ha tenido un efecto perjudicial en el bienestar de los pueblos del tercer mundo. Tres multimillonarios de personas en el Norte hoy poseen más activos que el PNB combinado de todos los países menos desarrollados y sus 600 millones de personas El derecho internacional desempeña un papel crucial para ayudar a legitimar y sostener las estructuras y procesos desiguales que se manifiestan en la creciente división entre el norte y el sur. De hecho, el derecho internacional es el principal lenguaje en el que la dominación se va a expresar en la era de la globalización, que está desplazando a los sistemas legales nacionales en su importancia, y, está teniendo un impacto sin precedentes en la vida de la gente común, armado con los poderes de las finanzas internacionales e instituciones comerciales para hacer cumplir una agenda neoliberal, el derecho internacional hoy amenaza con reducir el Estado a un simple ente distributivo social y supeditado al mercado, sin mayor injerencia en los asuntos económicos y cooptado por las directrices del derecho internacional privado.

Por último, tenemos lo que concierne a la teoría de los derechos humanos y la documentación oficial sobre tratados y convenios firmados por Colombia desde 1984 hasta el 2018, lo que atañe a las relaciones internacionales desde la mirada crítica de los TWAIL. Si se observa detenidamente la historia de reciente de los países del Tercer Mundo, puede intuirse que, incluso el discurso internacional sobre los derechos humanos está siendo manipulado para promover y legitimar los objetivos neoliberales. En resumen, la independencia económica y política del tercer mundo está siendo socavada por políticas y leyes dictadas por el primer mundo y las instituciones internacionales que controla. Antes de continuar con otros autores que serán mencionados en el Estado del Arte, que reproduzco a continuación, se esboza un mapa conceptual sobre la epistemología del Derecho Internacional y TWAIL. 


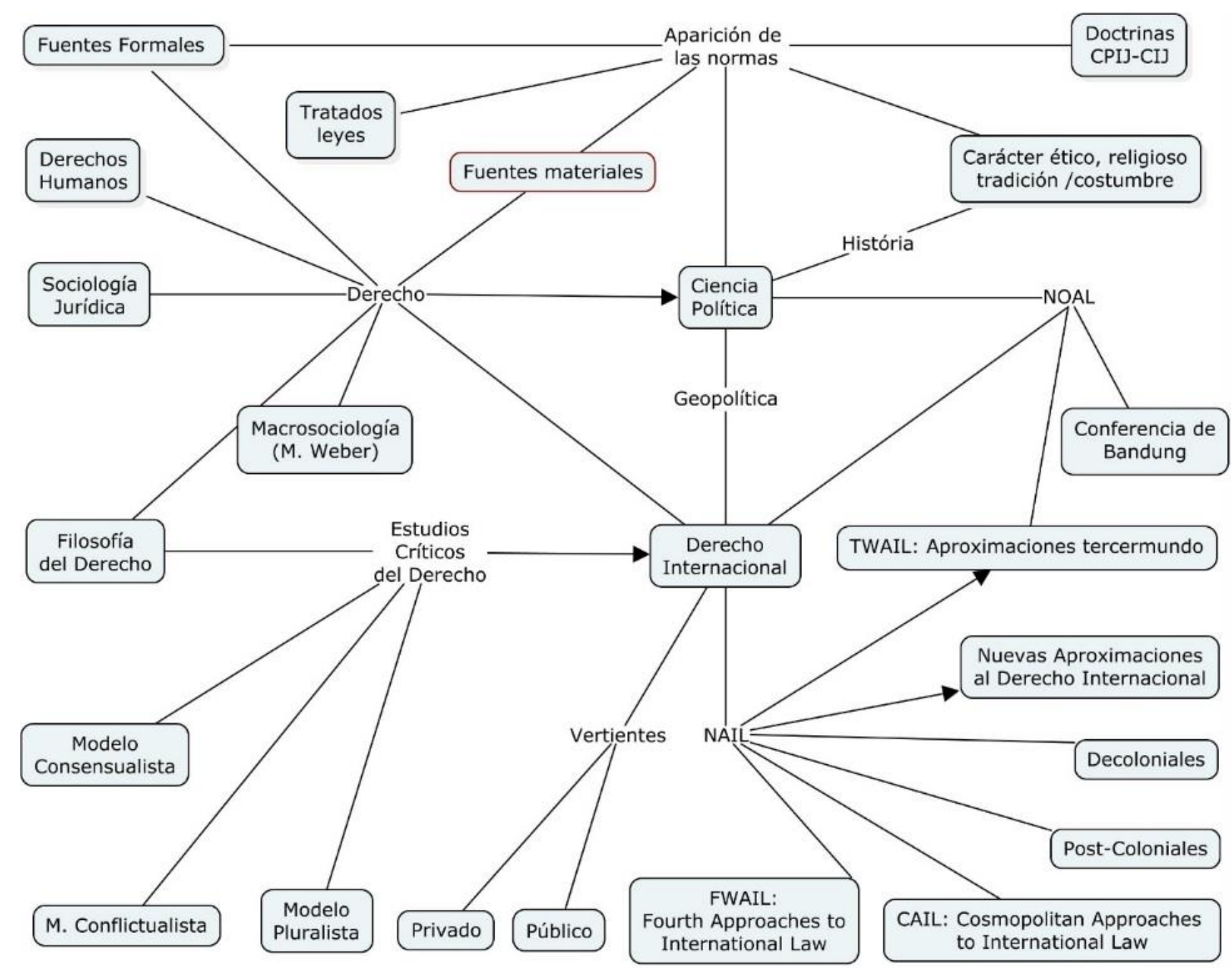

Figura 2: aproximación a la epistemología del Derecho Internacional y las teorías.

Fuente: tomado de Jorge Diego Megía Cortez (2020).

Desafortunadamente, TWAIL (enfoques del tercer mundo para el derecho internacional) no ha tenido la capacidad de criticar efectivamente el derecho internacional privado, abiertamente neoliberal o proyectar una visión alternativa del derecho internacional público, dominado ideológicamente por los académicos del norte. Siguiendo por la ruta de los académicos clásicos TWAIL tenemos en primer lugar a Sundhya Pahuja con "la poscolonialidad del derecho internacional" un texto escrito para el Harvard International Law Journal en el 2005, que aborda la relación entre el derecho internacional y el imperialismo.

\section{Estado del arte o de la cuestión}

Para la realización de este sondeo teórico o Estado del Arte preliminar, se tuvieron en cuenta, tanto los elementos básicos del Derecho Internacional como los documentos clave de los teóricos TWAIL, su resultado se agrupa en tres filtros, el primero autores/teóricos nacionales, el segundo filtro está constituido por: tesis, artículos académicos y resultados de investigación 
y el tercer filtro por otras fuentes generales. En el rastreo realizado a las bases de datos de las universidades nacionales (primer filtro) se pudo constatar que, únicamente en la Universidad de los Andes y en El Externado, en cabeza del profesor José Manuel Barreto, se encontraron artículos, tesis o textos relacionados con las teorías TWAIL. De este académico se extrajeron tres artículos fundamentales que son en su orden:

1) "Human Rights from a Third-World Perspective: Critique, History and International Law" (Derechos humanos desde una perspectiva del Tercer Mundo: crítica, historia y derecho internacional) 2) Eurocentric and Third-World Histories of Human Rights (Historias eurocéntricas y del tercer mundo sobre los derechos humanos) 3) Decolonial Thinking \& Human Rights (Pensamiento decolonial y derechos humanos) 4) Epistemologies of the South and Human Rights: Santos and the Quest for Global and Cognitive Justice (Epistemologías del Sur y los Derechos Humanos: Boaventura de Sousa Santos y la búsqueda de la justicia global y cognitiva) este último de una pertinencia esencial para el objeto de estudio en cuestión, por cuanto se pretende una aproximación a Latinoamérica. En otros buscadores tales como, Google academics, Scielo, Dialnet, Redalyc y el Tesauro de la Unesco, se encontraron también, artículos de revistas, producto de trabajos de grado e investigaciones de grupo, la gran mayoría en inglés, algunas en portugués y solo una en español. De estas se extrajeron los siguientes títulos (segundo filtro).

a) Third World Approaches to International Law and human rights: some considerations publicado en la Revista de Investigações Constitucionais, un estudio publicado por Larissa Ramina (2018), en el marco de un postdoctorado realizado entre la Universidad de Paris Ouest Nanterre y la Universidad Federal de Paraná y que, como su nombre lo indica realiza algunas consideraciones y críticas al modelo TWAIL desde la óptica del investigador.

b) En español, se encontró una tesis de grado del doctorado en Derecho y Ciencias Políticas de la Universidad de Barcelona titulada Hacia una reconsideración del prohibicionismo de las drogas en el derecho internacional desde el tercer mundo TWAIL escrita por José Manuel Luque González (2017) que también con una mirada crítica analiza los alcances de las perspectivas TWAIL no solo como países productores sino también como consumidores frente a las relaciones internacionales con los países del norte.

c) De otro lado, una tesis de maestría en desarrollo sostenible titulada: Sustainable Development in International Law and the protection of the Global Commons (2017) Este estudio examina la protección legal de los bienes globales comunes al enfocarse en el concepto de desarrollo sostenible, como se entiende en la Declaración de Principios de Derecho Internacional de Nueva Delhi sobre Desarrollo sostenible. La protección legal actual de tres de los cuatro acuerdos globales se analiza literalmente de lege lata (la ley tal como es). Luego, el estudio examina tres propuestas legales 
estipuladas dentro del derecho internacional, seleccionadas por su aplicabilidad para fortalecer la protección legal de los acuerdos globales que buscan la salvaguarda y la protección legal de los bienes, se examina el alcance de su incorporación al Desarrollo Sostenible y se analiza la proyección legal: de lege ferenda (la ley como debería ser).

d) Un artículo para una revista suiza de derecho internacional: "What Should Remain of the Critical Approaches to International Law? International Legal Theory as Critique (¿Qué debe quedar de los enfoques críticos del derecho internacional? Teoría jurídica internacional como crítica) escrito por Tilmann Altwicker \& Oliver Diggelmann, profesores de derecho internacional de la ciudad de Zurich, Suiza. (2014) El artículo es una especie de "estado del arte" desde una visión euro-centrista sobre los "enfoques críticos" del derecho internacional, cuando la noción de "enfoques críticos" se utiliza como un término general y se refiere a un conjunto de ideas poco relacionadas o supuestos, que incluyen los "nuevos enfoques del derecho internacional" (NAIL) o "Newstream", en contraste con los "Enfoques del Tercer Mundo al Derecho Internacional" (TWAIL) y una aproximación a los "enfoques feministas" desde ese Newstream.

Por último, (Tercer filtro) una serie de artículos y capítulos de libros escritos por reconocidos exponentes de la escuela TWAIL tales como: Meghna Kumar, B.S. Chimny, Makau Mutua entre otros, y que sin duda estarán insertos en el marco teórico de la presente investigación y que son en su orden:

1) What is TWAIL? (¿Qué es TWAIL?) De la profesora Meghna Kumar, a partir de un documento de Anthony Anghie, fundamental para entender la génesis de dichas teorías y sus principales autores, en su mayoría de origen asiático.

2) Un artículo de Timiebi Aganaba-Jeanty para la revista Space policy de la Universidad McGill, Instituto de Derecho Aéreo y Espacial. Quebec, Canadá titulado "Presentación de los enfoques cosmopolitas del derecho internacional (CAIL) un lente para analizar los problemas de gobernanza que afectan a los emergentes y aspirantes actores espaciales”. Este articulo habla sobre la libertad de exploración, libertad de uso (científica y actividades comerciales), la libertad de acceso al espacio ultraterrestre y la libertad de investigación científica en conformidad con el derecho internacional.

3) 3) Un artículo de B.S. Chimni, escrito para la International Community Law Review (2006) titulado: "Third World Approaches to International Law: A Manifesto" (Enfoques del Tercer Mundo al derecho internacional: Un manifiesto). En él se asume que, el proceso de globalización. ha tenido un efecto perjudicial en el bienestar de los pueblos del tercer mundo. Solamente tres multimillonarios en los países del Norte hoy poseen más activos que el PNB combinado de todos los países menos desarrollados y sus 600 millones de personas. El derecho internacional desempeña un papel crucial para ayudar a legitimar y sostener las estructuras y procesos desiguales que se manifiestan en la creciente división norte-sur. 
4) 4) "Newness, imperialism, and international legal reform in our time: a twail perspective" (Novedad, imperialismo y reforma legal internacional en nuestro tiempo: una perspectiva Twail), un artículo de Obiora Okafor para el Osgoode Hall Law Journal de la Universidad de Toronto (2005) Este artículo examina, desde un enfoque crítico del Tercer Mundo y las perspectiva del derecho internacional (TWAIL), la afirmación altamente consecuencial de que los ataques terroristas a los Estados Unidos el 11 de septiembre de 2001 inauguraron una nueva era en las relaciones internacionales tan radicalmente significativa, como una manera de justificar la reducción o el debilitamiento severo de ciertas normas fundamentales, de hecho constitucionales, de derecho internacional, tales como: la prohibición absoluta de la práctica de la tortura; la prohibición del uso unilateral de fuerza por los estados, establecido por el artículo 2 de la Carta de los Estados Naciones (la Carta de las Naciones Unidas); y la prohibición de ataques preventivos que no cumplen con los requisitos del artículo 51 de ese mismo tratado. Otros artículos relacionados fueron desestimados por ser considerados redundantes o poco importantes para el objeto de estudio en cuestión.

\section{Política exterior de Colombia en materia de Derechos Humanos9}

El siguiente párrafo traduce literalmente lo expresado por la Cancillería de la República de Colombia en su sitio web, se translitera con el ánimo de evidenciar cómo se muestra al mundo y será objeto de análisis en los párrafos subsiguientes.

Colombia es un país comprometido con la promoción, respeto y la garantía de los Derechos Humanos y las normas del Derecho Internacional Humanitario, con una institucionalidad fortalecida y con amplios espacios de diálogo que permiten la interlocución directa entre el Estado y las Organizaciones de la Sociedad Civil. Asimismo, cuenta con un avanzado marco normativo en la materia que ha servido de modelo a otros países, además ha ratificado la mayoría de los instrumentos internacionales relativos a los Derechos Humanos y DIH, los cuales honra en su seguimiento y cumplimiento de obligaciones.

Así, la Constitución de Colombia prevé que los tratados y convenios internacionales ratificados por el Congreso, que reconocen los derechos humanos y prohíben su limitación en los estados de excepción, prevalecen en el orden interno. De esa manera, los derechos y deberes consagrados en la Carta Política se interpretan de conformidad con los tratados internacionales sobre derechos humanos ratificados por Colombia.

Bajo el liderazgo del presidente Iván Duque, el Ministerio de Relaciones Exteriores se ha fijado como uno de sus objetivos el de participar responsable y sosteniblemente en los escenarios internacionales, especialmente de carácter multilateral, en defensa de la seguridad y la estabilidad internacionales, privilegiando el compromiso con la democracia representativa y el Estado de Derecho.

9 Citar de la página: http://nuevayork-onu.mision.gov.co/politica-exterior-colombiana-materia-derechoshumanos 
Con ese fin, la Misión de Colombia ante Naciones Unidas realiza acciones ante ese organismo a través de la promoción de relaciones respetuosas y responsables con actores del Sistema Internacional en materia de derechos humanos, privilegiando la transparencia y el intercambio de buenas prácticas en su promoción y protección.

Lo anterior, permite a su vez apoyar el ejercicio de los derechos fundamentales, sociales, económicos y culturales definidos en la Constitución Política de Colombia

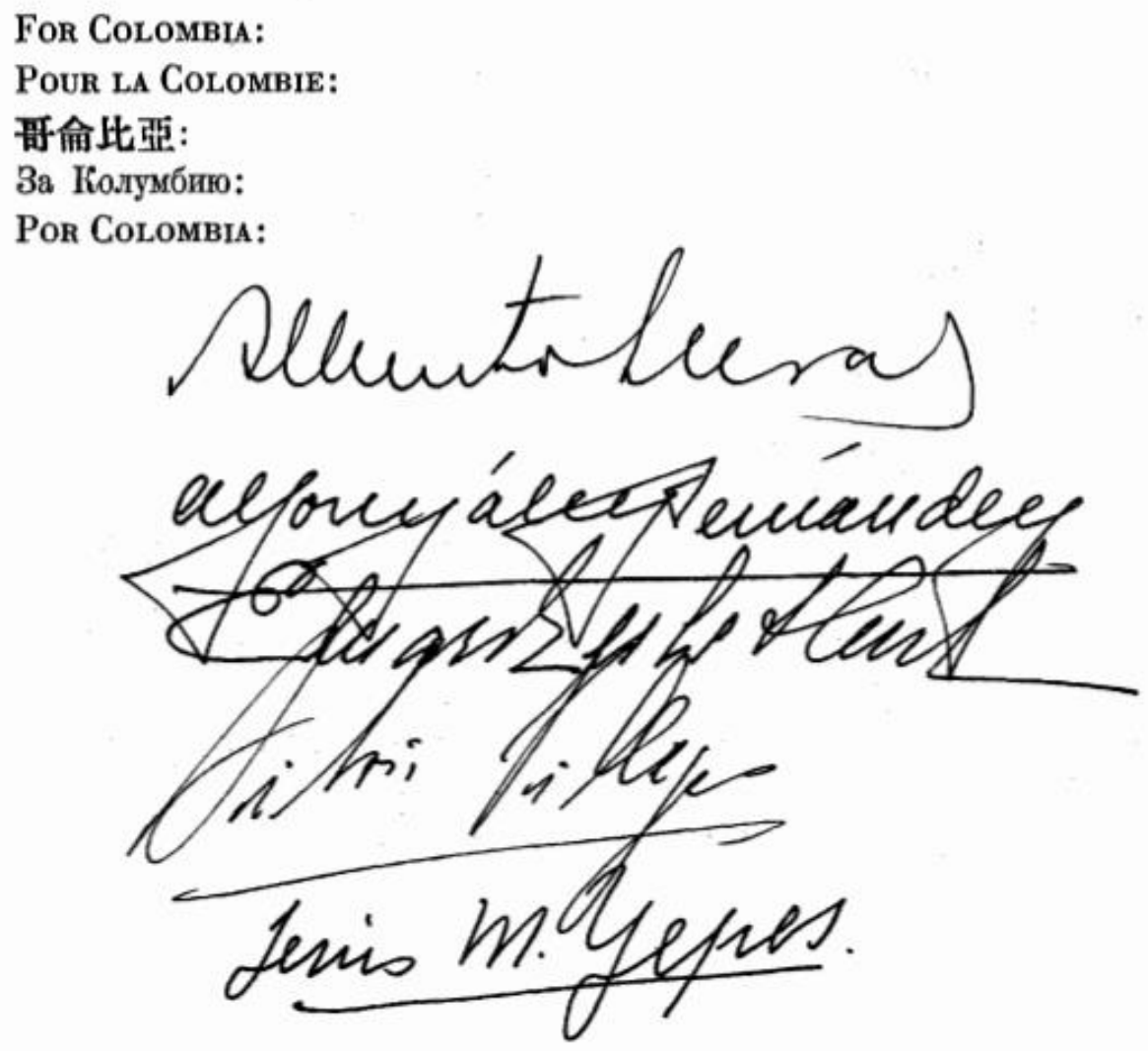

Figura 3: firma del representante colombiano en la Carta de San Francisco.

Fuente: Cancillería de Colombia.

\section{REFERENCIAS BIBLIOGRÁFICAS}

Aganaba-Jeanty, T. (2016). Introducing the Cosmopolitan Approaches to International Law (CAIL) lens to analyze governance issues as they affect emerging and aspirant space actors. Space Policy, 37, 3-11. https://doi.org/10.1016/j.spacepol.2016.05.007

Altwicker, Tilmann; Diggelmann, Oliver (2014). What should remain of the critical approaches to international law? International legal theory as critique. Schweizerische Zeitschrift für internationales und europäisches Recht (SZIER), 24(1):69-92.

Austin, Sarat. 2001. "El derecho está en todas partes: el poder, la resistencia y la conciencia jurídica de los pobres que viven de la asistencia social" En: Mauricio García (editor). 
Sociología jurídica: teoría y sociología del derecho en Estados Unidos. 217 - 266. Bogotá, Universidad Nacional de Colombia.

Barreto, J-M. (2015) Six Books: International Law, Human Rights and the Politics of the Turn to History. Critical Legal Thinking. Law \& the Political, 27 March 2015 Tomado de: https://criticallegalthinking.com/2015/o3/27/six-books-international-law-humanrights-politics-turn-history/

Barreto, José-Manuel. (2018) ¿Qué es TWAIL? Un estudio de las Aproximaciones Tercermundistas al Derecho Internacional. Pp. 1-13. Tomado de: https://icrp.uexternado.edu.co/wpcontent/uploads/sites/4/2018/o6/Barreto_Proyecto_TWAIL.pdf

Browlie, I. (1995) "Principles of International Public Law" Clarendon Press. Londres, pág. 2324

Carrera Damas. Germán (1930) «La República de Colombia y el nacimiento del proyecto americano de Bolívar: Causas de la disolución de la Gran Colombia». Banco de la República. Tomado de: https://www.banrepcultural.org/bibliotecavirtual/credencial-historia/numero-124/la-republica-de-colombia-y-el-nacimientodel-proyecto-de-bolivar

Chimni, B. (2003). 'Third World Approaches to International Law: A Manifesto' in Antony Anghie et al (eds), The Third World and International Order: Law, Politics and Globalization. 47

Chimni, B. (2007). A Just World Under Law: A View from the South, 22 AM. U. INT'L L. REV. 199.

De la Torre, Jesús Antonio. (2007) Apuntes para una introducción filosófica al Derecho. Capítulo 5. Marxismo. México, Editorial Porrúa. Pp. 103-127

Durkheim, Emile. (2001) La división social del trabajo. Pp. 36-102.

Erazo Coral, María Elena (2008) “Construcción de la nación colombiana”. Revista Historia de la Educación Colombiana $\mathrm{N}^{\mathrm{o}} 11$. Pasto, Nariño. Pp. 33-52

Fariñas D. María J. (1988) Tensión y distinción entre la Sociología del Derecho y la Dogmática jurídica en la obra de Max Weber: un problema metodológico. P. 9 Madrid

Galanter, Marc. (2003) "Por qué los "poseedores" salen adelante: especulaciones sobre los límites del cambio jurídico. En, García, Mauricio. Sociología Jurídica. Teoría y sociología del derecho en Estados Unidos. Universidad Nacional de Colombia. Bogotá. Pp. 69-103.

López Medina, Diego Eduardo (2004) Teoría impura del derecho. La transformación de la cultura jurídica latinoamericana, Bogotá, Universidad de los Andes, Legis, Universidad Nacional de Colombia.

López Zea, Rubén D. (1988) Política Internacional. $2^{\text {a }}$ Edición. Ed. Copiyepes. Págs 252-253 Luque González J.M. (2017) Hacia una reconsideración del prohibicionismo de las drogas en el derecho internacional desde el Tercer Mundo (TWAIL) Universitat de Barcelona. Págs. 1-289

Okafor, Obiora Chinedu. "Newness, Imperialism, and International Legal Reform in Our Time: A Twail Perspective." Osgoode Hall Law Journal 43.1/2 (2005): 171-191. Tomado de: http://digitalcommons.osgoode.yorku.ca/ohlj/vol43/iss1/7 
Pahuja, S. Harvard international law journal, ISSN 0017-8063, Vol. 46, No 2, 2005, págs. 459-470

Rajagopal, Balakrishnan. el Derecho Internacional desde Abajo (ILSA, 2010).

Rodríguez Garavito, César. (2011) Un nuevo mapa para el pensamiento jurídico latinoamericano (Introducción). En: Rodríguez Garavito, César (Coord.) El derecho en América Latina. Un mapa para el pensamiento jurídico del siglo XXI. Buenos Aires, Siglo XXI. Pp. 11-17.

Sarat, Austin. (2001) “El derecho está en todas partes”: el poder, la resistencia y la conciencia jurídica de los pobres que viven de la asistencia social. En García Villegas, Mauricio (Ed). Teoría y Sociología del Derecho en Estados Unidos. Universidad Nacional, Bogotá. 2001. Pp. 219-266.

Sarenmalm, I., (2017): Sustainable Development in International Law and the protection of the Global Commons. Master thesis in Sustainable Development at Uppsala University, No. 2017/29, $31 \mathrm{pp}, 30$ ECTS/hp

Sauvy, Alfred (1952). “Trois mondes, une planète”, L'Observateur, 14 de agosto de 1952, nº18, pág. 14. Tomado de: http://www.hommemoderne.org/societe/demo/sauvy/3mondes.html

Souza, M. L. (2001). Introducción: El Uso Alternativo del derecho. Génesis y evolución en Italia, España y Brasil. Bogotá: Ilsa, Universidad Nacional de Colombia. pp. 4-40.

Sorensen, Max, Manual de Derecho Internacional Público, Fondo de Cultura Económica, 1994, p. 151

Wallerstein, Immanuel (Coordinador). 2006. Abrir las ciencias sociales. Informe de la Comisión Gulbenkian para la reestructuración de las ciencias sociales. Siglo XXI editores.

Weber, Max. (1922) Economía y sociedad. Esbozo de sociología comprensiva. Pp. 1-29

\section{Cibergrafía}

Biblioteca Virtual de Derechos Humanos y Derecho Internacional Humanitario. Instrumentos Internacionales. Ministerio de Relaciones Exteriores. Tomado de: http://gruposmre.cancilleria.gov.co/sitios/DVAM/DIDHD/Biblioteca/SitePages/Ins trumentos\%20Internacionales.aspx

Colombia y los tratados internacionales de derechos humanos. Tomado de: http://epn.gov.co/elearning/distinguidos/DDHH/23_colombia_y_los_tratados_int ernacionales_de_derechos_humanos_la_pirmide_normativa.html

El Espectador (febrero 27 de 2020) "Es una intromisión a la soberanía": Duque sobre informe de Naciones Unidas Recuperado de: Tomado de: https://www.elespectador.com/noticias/politica/es-una-intromision-la-soberaniaduque-sobre-informe-de-naciones-unidas-articulo906687?fbclid=IwARoZtwucoiHeZ9vegWKSQca7B9kctroQ9oz_KaTP9H65ngcQ3_ Gjd77jVOA

El tiempo (mayo 19 de 1991) ¿Hacia dónde debe mirar Colombia? Tomado de: Disponible en: https://www.eltiempo.com/archivo/documento/MAM-86066 
Los defensores de los derechos humanos en Colombia son un "blanco fácil" Tomado de: Noticias ONU: Tomado de: https://news.un.org/es/story/2018/12/1446951

Política exterior colombiana en materia de derechos humanos. Tomado de: Cancillería de Colombia. Disponible en: http://nuevayork-onu.mision.gov.co/politica-exteriorcolombiana-materia-derechos-humanos

Sierra Camargo, Jimena. (2017) La importancia de decolonizar el derecho internacional de los derechos humanos: el caso de la consulta previa en Colombia. Revista Derecho del Estado. Universidad Externado de Colombia. Disponible en: https://uexternado3.metarevistas.org/index.php/derest/article/view/5042

\section{Artículos, Leyes y Decretos}

Convención sobre la tortura y otros tratos o penas crueles inhumanos o degradantes Disponible en: http://apw.cancilleria.gov.co/tratados/SitePages/VerTratados.aspx?IDT=98900981$5 \mathrm{a} 8 \mathrm{a}-4 \mathrm{bcb}-97 \mathrm{f} 2-9 \mathrm{~d} 36 \mathrm{f} 85 \mathrm{afc} 83$

Decreto 768 de 1988. (abril 26). Por el cual se promulgan unos tratados internacionales. Tomado de: Sistema Único de Información Normativa (SUIN) Disponible en: http://www.suin-juriscol.gov.co/viewDocument.asp?ruta=Decretos/1162370

Ley 70 de 1986 - Colombia. (diciembre 15) Tomado de: Lex Base. Disponible en: https://www.lexbase.co/lexdocs/indice/1986/loo7ode1986\#: :text=Ley\%2070\%20 de\%201986\%20\%2D\%20Colombia,el\%2oDecreto\%20768\%20de\%201988. 